\title{
Two-Steps Slotting Method in Magnet Edge of PMG for Wind Energy Harvesting
}

Tajuddin Nur ${ }^{1}$, Marsul Siregar ${ }^{1 *}$

${ }^{1}$ Department of Electrical Engineering, Faculty of Engineering, Atma Jaya Catholic University of Indonesia, Jl Sudirman No 51 Jakarta 12930, Indonesia

\begin{abstract}
This paper focuses on the study of how to decrease the value of the cogging torque in an Inset Permanen Magnet Generator. The permanent-magnet generator of an integral slot number with 24 slots and 8 poles was selected and investigated for the purposes of this study. The cogging torque of the permanent-magnet generator can be minimized by combining two slottings in the magnet edge with a gradually inclined surface end. The electromagnetic simulations and analysis of the permanent-magnet generator studied were performed using numerical analysis by means of FEMM 4.2. To obtain a faster computation, FEMM 4.2 was combined with LUA 4.0 programming. Using FEMM 4.2, it was found that by applying two-step slotting in the magnet edge combined with a gradually inclined surface end, the cogging torque of the permanent-magnet generator selected can effectively reduce the cogging torque of the machine by approximately $98.14 \%$ compared against the initial magnet model. It can be concluded that the integration of the two-step slotting in the magnet edge and the gradually inclined surface end can effectively reduce the cogging torque of the permanent-magnet generator machine. Furthermore, decreases in the density of the magnetic flux in the core of the permanent-magnet generator can also be found.
\end{abstract}

Keywords: Cogging torque; Finite element; Integral slot number; Permanent-magnet generator

\section{Introduction}

The application of permanent-magnet generators (PMGs) in our daily lives is now becoming more attractive and can be used with robotics, electric vehicles, wind turbines, and many other applications. Any PMG has some merit over other types of electrical machines. Wind power generation has recently been increasing in popularity because it is clean, has a low production cost, is abundant, and because market volume has tended to significantly increase compared with other energy sources. Using PMGs in renewable energy systems has benefits including high performance and efficiency, simplicity, and reliability of construction. However, the most important issue for PMG applications is the presence of cogging torque (CT). The CT in any PMG is impacted by the interaction among the magnetic flux force from the rotor core and stator teeth or stator slot in the stator core of the machine. The CT in electrical machines and in PMGs can produce noise, vibration, and many other complicated conditions that might appear in the machine application system.

Over the last few years, much research has addressed these issues and sought to reduce

${ }^{*}$ Corresponding author's email: marsul.siregar@atmajaya.ac.id, Tel: +62-215708826;

Fax: +62-215790057343

doi: 10.14716/ijtech.v11i7.4554 
and overcome the CT of Permanent Magnet Synchrounous Machine. Furthermore, many techniques for CT reduction have been proposed and developed worldwide; these reports are well documented (Chen et al., 2010; F.Scuiller, 2014; Zhou et al., 2015). Other CTdecreasing techniques have been reported in research, such as using a fractional slot number and phase, optimizing the magnet pole arc (Chen et al., 2010; F.Scuiller, 2014; Zhou et al., 2015), skewing stator slots or magnets (Chabchub et al., 2012; Ling and Nur, 2016), optimizing the stator slot opening width (Bianchi and Bolognani, 2002), applying a dummy slot in the stator core (Chabchub et al., 2012), and shifting magnet slotting (Chen et al., 2010 ) at the magnet edges of the Permanent Magnet Synchronous Machine (Dosiek and Pillay, 2007). However, the most effective technique for lessening the CT in PMG is combining two-step slotting with shaping the magnet edge in terms of a gradually inclined surface end (GISE). Two-step slotting at the magnet edge and shaping the magnet on the magnet surface provides more frequent interactions between the magnet fluxes in the rotor core and the stator slot opening of the machine.

The purpose of this paper is to propose a new technique for CT reduction in any PMG and to prove that employing the combination of TSS in the magnet edge with GISE in the magnet edge could effectively lessen the peak CT value in any PMG. The new technique of CT reduction can be applied to both integral slot numbers and fractional slot numbers; however, in this work, the analysis of PMGs focuses on the integral slot number. The authors have selected a PMG structure with 24 slots and 8 poles. Figure 1 illustrates the magnet structure of the PMGs, which is the initial structure. Figure 3a shows one-step slotting (OSS), and the Figure 3b presenst TSS. From Figure 1 it can be observed that the initial magnet structure is a conventional magnet structure, which means no slotting was applied in the magnet structure of the machine. Figure 3a demonstrates the use of OSS on the magnet edge, while Figure $3 \mathrm{~b}$ shows the application of TSS on the magnet edges of the PMG. All PMG structures have been studied and contrasted in this paper. The three structures for a 24 slot/8 pole of PMG are analyzed and compared in this paper.

In general, the magnet rotor in any PMG is similar to that used in surfaced-mounted permanent-magnet rotors. The difference between the PMGs studied in this paper and a surface-mounted machine is the iron tooth or rotor teeth within the rotor core. As a surface-mounted machine, the permanent magnets in the rotor core of the machine are the main causes of the magnetix flux in a PMG. In the PMG structures studied, the magnets of the machine are buried in the rotor core, leading to a compact and strong rotor construction compared against surface PMGs. The structures of PMGs proposed in this study are shown in Figure 3b.

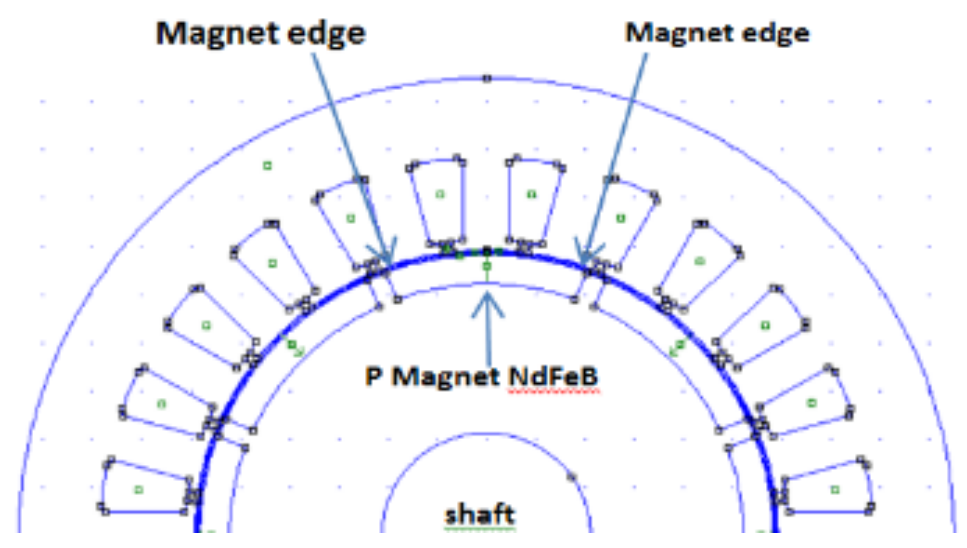

Figure 1 Construction of the Initial Structure (IS) of PMG 


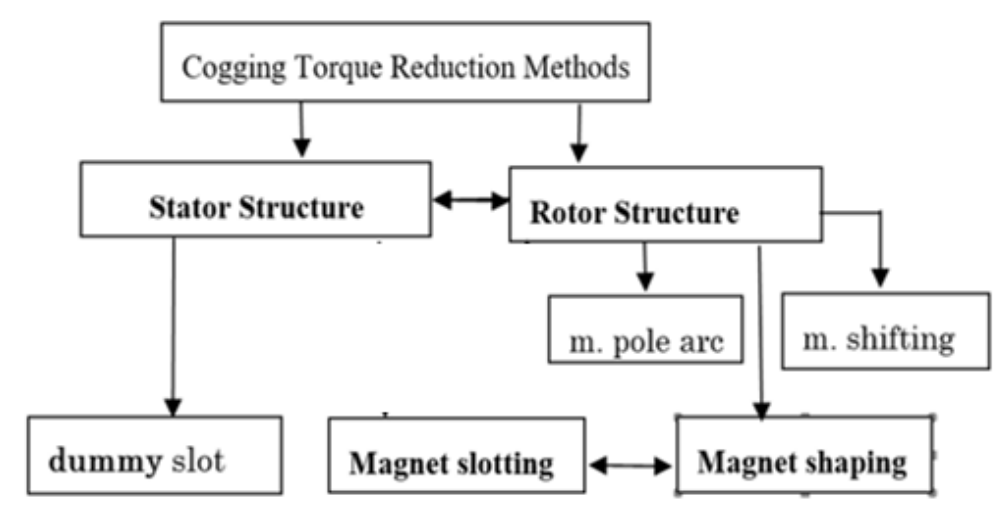

Figure 2 Classification of cogging torque (CT) reduction techniques for PMGs

\section{Methods}

This study investigates and compares three PMGs with different magnet structures. The initial magnet structure and the proposed PMG model are depicted in Figure 1. The performance of the PMG studied has been computed and analyzed using numerical analysis by means of finite element method magnetics (FEMM). Our research uses FEMM version 4.2. For the sake of increasing the speed of calculation processing, FEMM 4.2 was coupled with LUA 4.0 programming.

\subsection{Slotting Steps}

Slotting the magnet structure of any electrical machine or PMG is one of the CT reduction techniques applied in electric machine design and has been proposed and reported worldwide. The step of slotting is extended from existing CT reduction techniques previously proposed by researchers (Chen et al., 2010; F.Scuiller, 2014; Zhou et al., 2015) and other reports worldwide. Investigated the effect of slotting the magnet surface for the inner rotor type of permanet magnet machine, while F.Scuiller (2014) studied the influence of slotting the magnet surface for the inner rotor. Chen et al. (2010) studied the effect of slotting the magnet surface for the outer rotor type of fractional slot number on the CT reduction of a machine. Magnet edge shaping can increase machine performance and reduce the CT of a machine. Zhou et al. (2015) studied the effect of OSS on the magnet edge. The CT reduction techniques developed and proposed in this study combined the gradually inclined magnet surface with the slotting. Ling and Nur (2016) studied the impact of the slotting technique as a two-step slotting method in the magnet edge.

For the purposes of this study, three PMGs of Integral Slot Number (ISN) with different magnet structures of generic machines are investigated, compared, and presented. For the sake of simplicity, the saturation in both the stators and the rotor core of the PMGs studied is neglected. In addition, the leakage flux along the axial of the PMGs studied is not studied in detail. The magnetic flux orientation was assumed to flow radially along the axial length of the machine. The schematic of the CT reduction technique for PMG used in this study is shown in Figure 2. In Figure 2, one can observe that CT in PMG could be minimized by optimizing the stator structure or rotor structure or by combining the optimizations for both the stator and rotor core. This work presents the effectiveness of TSS in the magnet edge combined with GISE for the inner rotor permanent magnet machine of the generic machine. In this study, a 2D finite element method of FEMM 4.2 has been applied to analyze the performance of the PMMs. 


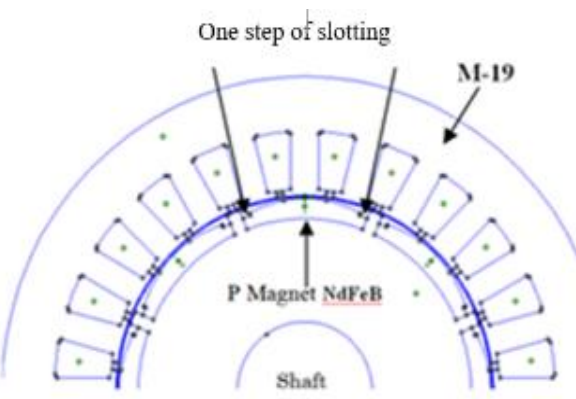

(a) One-step slotting

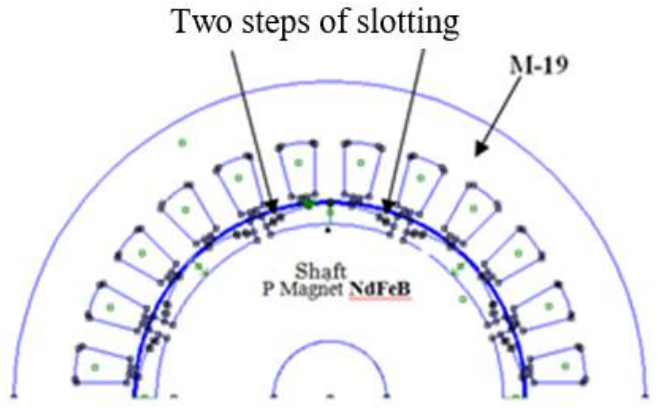

(b) Two-step slotting

Figure 3 One-step slotting (OSS) and two-steps slotting (TSS) techniques construction

The slotting steps at the magnet edge in Figures $3 \mathrm{a}$ and $3 \mathrm{~b}$ are based on the magnet structure of the PMG as illustrated in Figure 1. For Figures $3 \mathrm{a}$ and $3 \mathrm{~b}$, both sides of the magnets are slotted and shaped gradually. Thus, the magnet height of the initial structure is different from the magnet edge or the magnet of the machine. In addition, the slotting at the magnet edge increases the nodes on the magnet edge. This is essential for increasing the CT frequency of the machine and reducing the amplitude of CT. The nodes in the magnet edge are connected by a segment that provides a new flux path of the magnet into the machine's air gap.

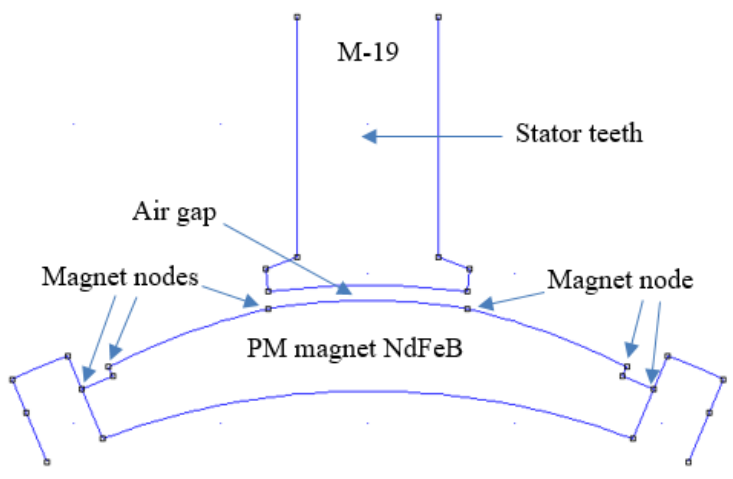

(a) One-step slotting

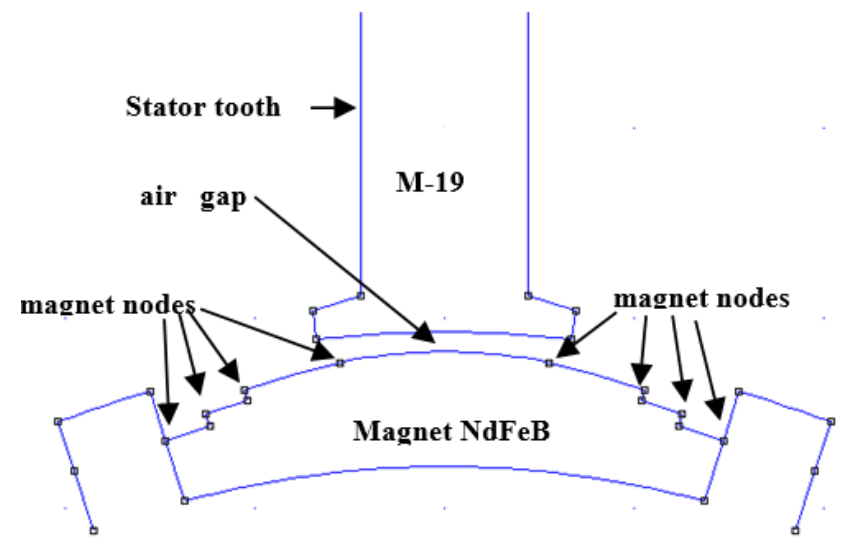

(b) Proposed two-step slotting

Figure 4 Detailed structures of one-step slotting (OSS) and two-step slotting (TSS) on the magnet edge.

\subsection{Effect of Two-Step Slotting of Magnet Edge}

Figure 4 demonstrates the effect of TSS on CT reduction for the proposed PMG. Figure $4 \mathrm{~b}$ shows TSS on the magnet edge; where the distribution of the magnetic flux in the machine core is discussed in this paper. The existence of TSS on the magnet edge can gradually reduce the distance between the magnet edge and the stator core of the machine. In addition, by applying TSS on the magnet edge, the magnet structures are divided into three layers: an upper layer, middle layer, and base layer. In the upper layer of the magnet (the nearest to the stator core), the magnet surface or the magnet edge is closest to the stator core. The magnet in this upper layer contributes most to directing magnet flux into the air gap and the slot opening width in the stator teeth. The distance between the magnet and the stator teeth is $1 \mathrm{~mm}$, while the slot opening width at the core of the stator is $2 \mathrm{~mm}$. Since the edge of the magnet is shaped gradually, the distance between the stator core and the magnet surface at one- and two-step slotting becomes larger compared with the upper layer closer to the stator teeth. At the base layer, the distance between the magnet edge and the stator core is the largest. The greater the depth of slotting and the length of slotting in 
the edge of the magnet, the larger the distance between the magnet edge and slot opening. This means that the magnetic flux density to reach the slot opening also decreases. This reduces the total magnetic flux flowing into the air gap of the machine.

Furthermore, a slotted magnet edge can increase the number of nodes in the magnet structure or in the magnet edge. The nodes on the magnet surface and magnet edge could be the basis of magnet flux flowing into the air gap of the machine. The following section compares normal flux and tangential flux density for all PMGs studied. Figures $5 a, 5 b$, and 5c, shows the tangential flux densities of the Initial Structure, One-step Slotting and the Two-steps Slotting, respectively. From Figure 5 a it can be observed that the peak tangential flux density of the initial structure is around 0.29 Tesla. After applying OSS to the magnet edge, the peak tangential flux density was reduced to 0.2 Tesla. Applying the combination of TSS and GISE of the magnet, the peak of tangential flux did not change much. However, the normal flux density profile of the machine tended to be sine compared with the PMG with OSS and the initial structure.

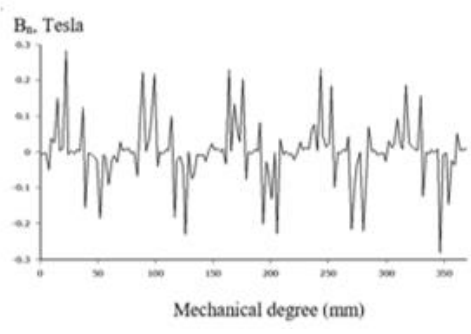

(a) Initial structure

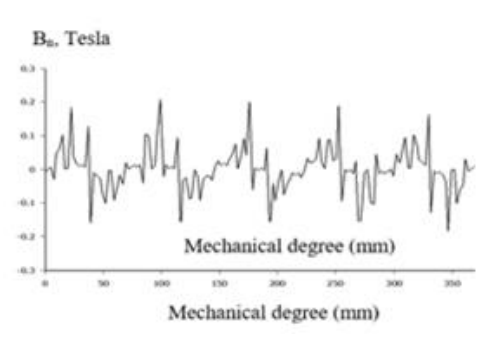

(b) One-step slotting

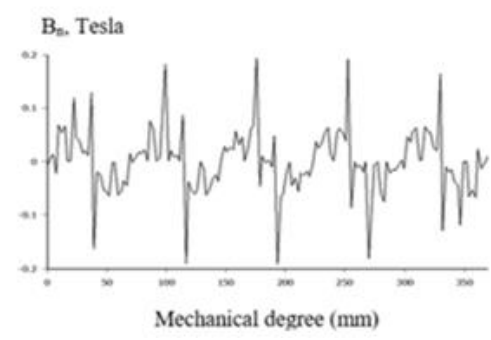

(c) Two-steps slotting

Figure 5 Tangential flux densities of IS, OSS and TSS of the PMG studied

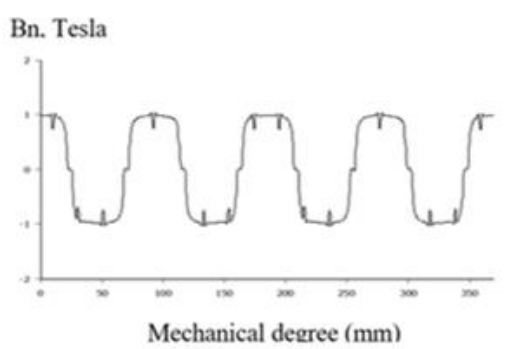

(a) Initial structure

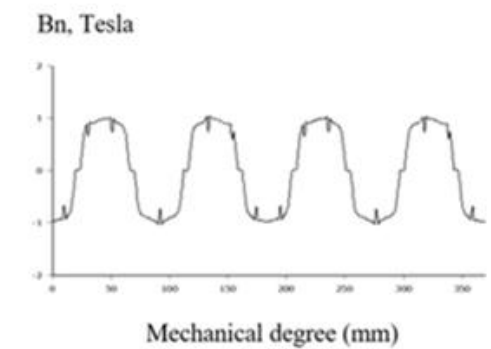

(b) One-step slotting

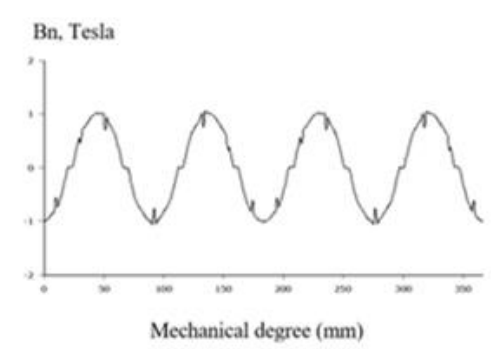

(c) Two-steps slotting

Figure 6 Normal flux densities for IS, OSS and TSS of PMGs studied

The normal flux densities for all PMGs studied are compared in Figure 6. Tangential flux density most contributes to increasing the CT peak of any machine. Thus, in order to minimize the CT of any electrical machine of PMG, the tangential flux density should be directly minimized. The best technique to reduce the tangential flux density in PMG is by optimizing the magnet edge by combining the TSS and GISE techniques on the magnet edge.

By applying TSS on the edge of the magnet, the density of tangential flux is greatly reduced compared to OSS and the initial structure. The presence of TSS on the magnet edge presents some nodes in the magnet edge. Each node has a coordinate position in $(x, y)$. The effects of nodes on the magnet edge are analyzed in this paper. The effective coordinate positions of magnet nodes denote $(\mathrm{x} 1, \mathrm{y} 1)$ and $(\mathrm{x} 2, \mathrm{y} 2)$. It found the value as $(-5.49566$, $57.6505)$ and $(5.49566,57.6505)$ from refrence $x, y$ of $(0,0)$ in the machine shaft. The coordinate positions of the nodes in the magnet are also presented in Table 1. It obtained that the different positions of gthe point at the magnet surface refere to the decraesed of CT. The magnet edge points are shown in Table 1. 
Table 1 The magnet edge points

\begin{tabular}{crrc}
\hline & $\mathrm{x}_{1}$ & $\mathrm{y}_{1}=\mathrm{y}_{2}$ & $\mathrm{y}_{2}$ \\
\cline { 2 - 4 } IS & 15.5431 & 56.1071 & 15.5431 \\
OSS & -5.36011 & 57.6489 & 5.36011 \\
TSS & -5.49566 & 57.6505 & 5.49566 \\
\hline
\end{tabular}

\section{Results and Discussion}

Finite element method magnetics version 4.2 (FEMM 4.2) was used to analyze the PMGs in this study. To increase the accuracy of the CT simulation results, some dummy lines were introduced in the machine's air gap. This paper used four dummy lines in the air gaps of all machines studied. As a consequence, the meshes and nodes of the machines studied increased dramatically, increasing simulation time compared to fewer dummy lines in the air gaps. Previous reports use FEMM software to analyze permanent-magnet performance, (Nur et al., 2020; Siregar et al., 2020). This software is user-friendly and very powerful for analyzing any complex structure of electric machines. The LUA 4.0 programming script was used to automate the virtual rotation of the magnet rotor and provide a virtual rotation. The stepping and the degree of mechanical rotation depended upon the input from the LUA 4.0 script.

On this research, the virtual rotation of the machine is $1^{\circ}$ (one degree) for every step of rotation. For an integral slot number of 24 slots and 8 poles, the total number of mechanical degrees for rotor rotation is $90^{\circ}$. Figure 4 reflects the different magnet rotor structures of PMGs studied in this paper. The initial structure seen in Figure 1a has two points at the magnet surface, while for one-and two-step slotting, the points are 6 and 8, respectively. The effect of the structure on the CT and density of the magnetic flux in the air gap was studied. To analyze the CT of the PMG studied, the authors used the Maxwell stress tensor method. The advantageous of this method, it requires only the local flux density distribution along a specific line or contour. In the simulation, the rotors of the PMGs studied are rotated using virtual rotation with small steps with $1^{\circ}$ for every degree of mechanical rotation. In addition, at each position of the rotor rotation of machines, the changing of the CT value in the machine air gap is calculated. Then, it can be reported that the CT peak value that has been expanded in the machine is the partial change in the CT peak value as concerns the virtual rotor movement. The value of the CT of PMGs is influenced by parameters in the machine structure, including the stack length of the machine, the radius of the rotor, normal flux density, and tangential flux density; however, the most important factors contributing to CT in machines are the normal and tangential flux density. The normal flux density of the magnet can produce normal force in the air gap of the machine, as formulated in Equation 1.

$$
F_{n}=\frac{1}{2 \mu_{0}}\left(B_{n}^{2}-B_{t}^{2}\right)
$$

where $F_{n}$ is the normal force, $B_{n}$ is the normal flux density, and $B_{t}$ is the tangential flux density. The normal force tends to distribute into the stator teeth of the machine; it may not contribute directly to the CT value of the machine. Another magnet flux density in the air gap of a machine is tangential flux density. It generates a tangential force $\left(F_{t}\right)$ in the air gap of the machine, as can be formulated in Equation 2.

$$
F_{t}=\frac{1}{\mu_{0}} B_{n} B_{t}
$$


The tangential force tends to flow into the slot opening in the stator core and generates CT in the air gap of the machine. The relation between tangential force and CT in the air gap of the machine can be formulated as follows;

$$
T_{c}=L_{s t k} \int_{0}^{2 \pi} F_{t} r^{2} d \theta \quad \text { or } \quad T_{c}=L_{s t k} \int_{0}^{2 \pi} r^{2} \frac{1}{\mu_{0}}\left(B_{n} B_{t}\right) d \theta
$$

Then, the formula can be written as Equation 3.

$$
T_{c}=\frac{L_{s t k}}{\mu_{0}} \int_{0}^{2 \pi} r^{2} B_{n} B_{t} d \theta
$$

where $T_{c}$ is cogging torque. In Equation 3, the CT value refers to the values of $B_{n}$ and $B_{t}$, but tangential flux density most contributes to the peak CT value. The reason is that tangential flux density tends to flow into the slot opening in the stator core and generate tangential force. The tangential force acting in the air gap of the machine can limit rotor rotation, particularly at low wind speed. As demonstrated by Equation 3, CT is affected by tangential force, so minimizing CT means minimizing tangential flux density. In order to minimize the tangential force to reduce the CT of the machine, the PMG structure should be modified or optimized; this modification can be done from the stator side, magnet rotor side, or both stator core and magnet rotor. In this work, tangential force is minimized by slotting the magnet edge of the machine. To reduce tangential force, TSS was selected. Reducing the Cogging Torque in any PMG by applying two-steps slotting to the magnet edge has been proposed and investigated by previous scholars. Ling and Nur (2016) used 24-slot, 8-pole structures for PMM; the air gap length of the machine was $2 \mathrm{~mm}$, and the slot opening width was $2 \mathrm{~mm}$. The CT of the machine was reduced by around 95\% compared with the initial model. In our work, the air gap length of the machine was $1 \mathrm{~mm}$, while the slot opening width was $2 \mathrm{~mm}$. The parameters related to the length and the height of the slotting of the magnet edge have been optimized using the response surface method. It is obtained that percentage of the CT decreasing of the proposed machine was $98.14 \%$ compared to the initial structure. The TSS technique is beneficial because it directly reduces tangential flux density. It also reduces the tangential flux density more effectively compared to the applied distributed slotting on the magnet surface (Chen et al., 2010; F.Scuiller, 2014). In addition, to improve the CT reduction of the proposed PMGs, the TSS technique developed in this work has been combined with GISE. As a result, CT is reduced more than achieved in past research (Ling and Nur, 2016). Another advantage of combining TSS and GISE in magnet edges was the significant reduction of tangential flux density; however, normal flux density reduction was not significant. The waveform of both normal flux density and tangential flux density of PMGs studied is presented in Figure 5 and Figure 6, respectively. Here, the tangential flux density most contributes to increasing the CT peak of any machine.

In this technique, the slot depth of the magnet from the rotor surface was fixed at 2.7 $\mathrm{mm}$, while the length of the slot magnet edge from the rotor teeth was also $2.7 \mathrm{~mm}$. For TSS, the magnet edge slotting depth was $3.3 \mathrm{~mm}$ from the surface of the rotor core. The length of the slot magnet was $2.7 \mathrm{~mm}$ and the slot was $2.5 \mathrm{~mm}$ from the rotor teeth. Since both sides of the magnet edge were slotted, the distance between the magnet edge and the slot opening width in the stator core increased. As a consequence, the magnet flux flowing into the air gap decreased. In addition, the TSS of the magnet edge of the proposed PMG achieves more point contact between the magnet edge or the magnet structure and the slot opening width in the stator core. This increases the machine's CT frequency, thereby significantly reducing the CT compared to the initial structure and OSS technique. Three PMGs with different magnet structures were investigated and analyzed using FEMM 4.2. The CT reduction of the PMGs studied is compared and presented in Figure 7. 


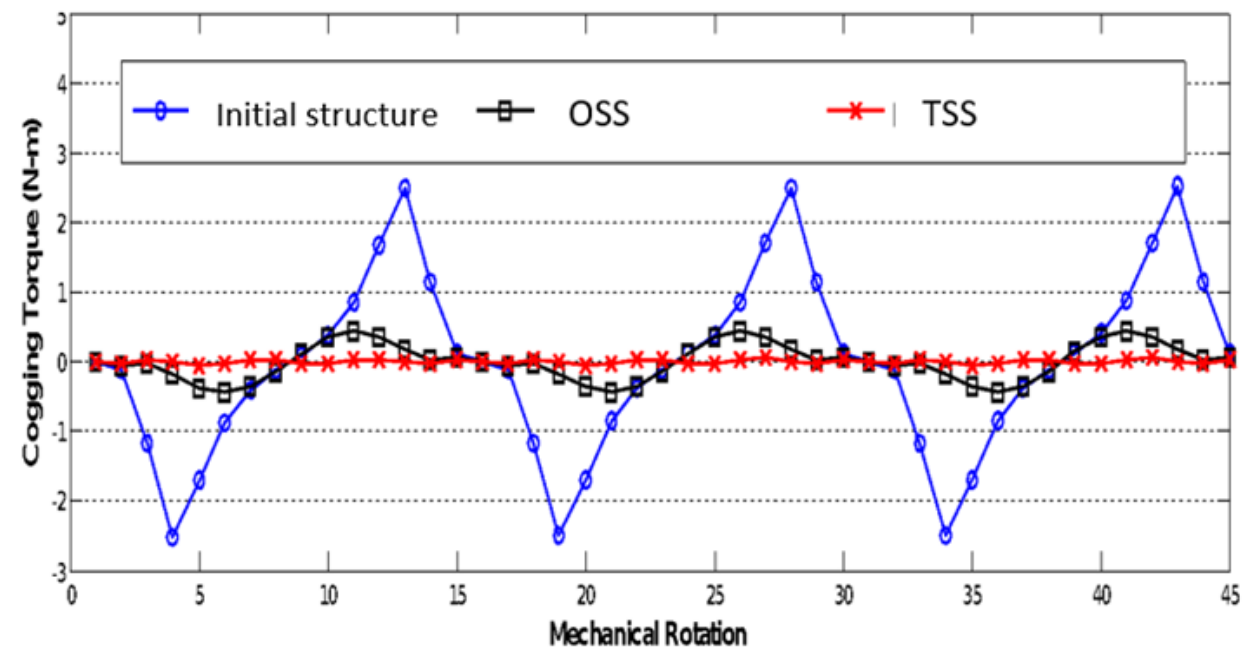

Figure 7 Cogging torque comparison for three Structured PMGs

Figure 7 compares the CT reduction for all PMGs studied. Here, for the structure of Figure 1, the initial structure of the PMG has the highest peak CT value (i.e., 2.5085881 Tesla). By employing OSS on the magnet edge, the CT of the machine was reduced to 0.4361457 Tesla. The CT of the PMG with OSS can be further reduced, as the CT reduction for the PMG with OSS is still insufficient for wind power applications. To further reduce CT, TSS and GISE should be applied to the magnet edge. By increasing the number of slots at the magnet edge using TSS and GISE, the CT of the proposed PMG can be reduced from 2.5085881 Tesla to 0.0465475 Tesla. So it can be stated that the value of the CT of the proposed of OSS and TSS method is lower than the CT value of PMG of the Initial sturcuture. For the TSS, the percentage of CT reduction for the proposed PMG with 24 slots and 8 poles is calculated as: $100 \%-[(0.0465475 / 2.50858810)] \times 100 \%=100 \%-1.86 \%=98.14 \%$.

\section{Conclusions}

This paper recommends combining TSS with GISE to reduce CT for the proposed PMSM. In comparison with the performance of the initial structure, the ability of the PMG to harvest electrical energy from wind can be improved by around $98.14 \%$. Using the FEMM, it was found that the CT of the PMG proposed as much as $98.14 \%$ in contrast with the PMG of the initial structure. It can be concluded that combining TSS and GISE effectively reduces the CT of a PMG. The combination of TSS and GISE on the magnet edge significantly decreased tangential flux. It can be concluded that combining TSS and GISE in PMGs enhances the energy harnessed from wind power.

\section{Acknowledgements}

Thank you to the Atma Jaya Catholic University especially the Engineering Faculty for supporting this research.

\section{References}

Bianchi, N., Bolognani, S., 2002. Design Techniques for Reducing the Cogging Torque in Surface Mounted Permanent Magnet Motors. IEEE Transaction on Industry Application, Volume 38(5), pp. 1259-1265 
Chabchub, M., Ben Salah, I., Krebs, G., Neji, R., Marchand, C., 2012. PMSM Cogging Torque Reduction: Comparison between Shape of Magnet. In: First International Conference on Renewable Energy Vehicle Technology, pp 206-211

Chen, N., Ho, S.L., Fu, W.N., 2010. Optimization of Permanent Magnet Surface Shape of Electric Motor for Minimization of Cogging Torque using FEM. IEEE Transaction on Magnetics, Volume 46(6), pp. 2478-2481

Dosiek, L., Pillay, P., 2007. Cogging Torque Reduction Permanent Magnet Machine. IEEE Transaction on Industry Applications, Volume 43(6), pp. 1656-1571

Ling, J.M., Nur T., 2016. Influence of Edge Slotting of Magnet Pole with Fixed Slot Opening Width on the Cogging Torque in Inset-Permanent Magnet Synchronous Machine. Advanced Mechanical Engineering, Volume 8(8), pp. 1-9

Nur, T., Joe, L.E., Siregar, M., 2020. Novel of Cogging Torque Reduction Technique for Permanent Magnet Generator by Compounding of Magnet Edge Shaping and Dummy Slotting in Stator Core. International Journal on Advanced in Science Engineering and Technology, Volume 10(3), pp. 1191-1199

Scuiller, F., 2014. Magnet Shape Optimization to Reduce Pulsating Torque for a Five-Phase Permanent Magnet Low Speed Machine. IEEE Transsaction on Magnetics, Volume 50(4), pp. 1-9

Siregar, M., Wohon, D.R., Nur, T., 2020. A New Technique to Reduce the Cogging Torque of Integral Slot Number in Permanent Magnet Synchronous Machine. International Journal on Advanced in Science Engineering and Technology, Volume 10(4), pp. 14361443

Zhou, Y.Y., Li, H., Meng, G., Shou, S., Cao, Q., 2015. Analytical Calculation of Magnetic Field and Cogging Torque in Surface-Mounted Permanent-Magnet Machines Accounting for any Eccentric Rotor Shape. IEEE Transaction on Industrial Electronics, Volume 62(6), pp. 3438-3447 